\title{
Clinical and Predictive Significance of Hyponatremia after Aneurysmal Subarachnoid Hemorrhage
}

\author{
Vladimir Vrsajkov ${ }^{1}$, Gordana Javanović ${ }^{1}$, Snežana Stanisavljević ${ }^{1}$, Arsen Uvelin², Jelena Panti Vrsajkov ${ }^{3}$ \\ ${ }^{1}$ Department of Anesthesia and Intensive Care, Clinical Centre of Vojvodina, Novi Sad, Serbia \\ ${ }^{2}$ Clinical Centre of Vojvodina, Emergency Centre, Novi Sad, Serbia \\ ${ }^{3}$ Health Care Centre "Novi Sad, Novi Sad, Serbia
}

\section{ABSTRACT}

Objective: Hyponatremia after SAH was the object of several studies with conflicting results. The aim of this study was to determine a predictive correlation of hyponatremia with delayed cerebral ischemia $(\mathrm{DCl})$ and poor clinical outcome.

Material and Methods: We have used a retrospective hospital chart review of 82 patients with SAH treated from August 2008 to August 2010 . Patients were divided into hyponatremia and normonatremia groups. Hyponatremia was defined as serum sodium level <135 mmol/I. Information compared and analyzed included demographics, preoperative neurological status, aneurysm characteristics, postoperative intensive care, duration of stay, DCl and clinical outcome at hospital discharge. $\mathrm{P}<0.05$ was considered significant.

Results: Thirty-two patients with SAH (39\%) developed hyponatremia. In that group we had a significantly higher WFNS score at admission ( $p=0.03$ ) and longer duration of stay in intensive care $(p=0.001)$. DCl with transit or definitive deficit included 20 patients $(62 \%)$ in the hyponatremia group, and 19 patients $(38 \%)$ in the normonatremia group $(p=0.03)$. Binary enter logistic regression revealed a significant correlation of hyponatremia with DCl $(p=0.03)$ and poor clinical outcome $(p=0.001)$.

Conclusion: This result revealed a possible use of hyponatremia as an additional predictor of developing DCl and poor clinical outcome.

Key Words: Hyponatremia, cerebral ischemia, subarachnoid hemorrhage, Glasgow outcome scale

Received: 05.12 .2011

Accepted: 16.05 .2012

\section{Introduction}

Patients with subarachnoid hemorrhage often develop hyponatremia during the first two weeks after the cerebral aneurysm rupture. Hyponatremia prevalence rates are approximately $30-55 \%$ (1-3). The etiology of hyponatremia is still unclear but it is probably mediated by hypothalamic injury secondary to SAH. This injury leads either to increased secretion of antidiuretic hormone, which causes the syndrome of inappropriate antidiuretic hormone (SIADH), or to Cerebral salt wasting (CSW) caused by enhanced release of atrial natriuretic peptide, brain natriuretic peptide, dendroaspis natriuretic peptide, adrenomedullin or noradrenaline (4-11). Regardless of the etiology, hyponatremia could deteriorate the neurological condition of SAH patients and severe hyponatremia increases the risk of mortality and morbidity (12).

Delayed cerebral ischemia (DCl) is a major cause of morbidity and mortality after aneurysmal subarachnoid hemorrhage. $\mathrm{DCl}$ is a term applied to any neurological deterioration (hemiparesis, dysphasia, altered consciousness) presumed related to ischemia that persists for more than an hour and cannot be explained by other physiological abnormalities noted on standard radiographic, electrophysiologic or laboratory findings (12-14).
The predictive value of hyponatremia on clinical outcome and correlations between $\mathrm{DCl}$ and hyponatremia was a subject of some studies but the results were inconsistent $(2,3,15-17)$. These results did not confirm or negate a possible connection, and our study is an attempt to provide a new contribution in resolving this potentially very interesting matter.

The aim of this retrospective study is to determine the incidence of hyponatremia after cerebral aneurysm rupture, clinical significance and predictive correlation with symptomatic cerebral vasospasm and poor clinical outcome.

\section{Material and Methods}

This retrospective study was approved by the Clinical centre of Vojvodina Ethics Committee. The medical records of all patients admitted to the Clinical centre of Vojvodina with spontaneous SAH between August 2008 and August 2010 were reviewed. All patients had subarachnoid hemorrhage diagnosed with computed tomography, and confirmed saccular aneurysm origin. Excluding criteria were 1) cerebral aneurysm of traumatic, fusiform or micotic origin. 2) death within 5 days after the SAH. 3) concomitant severe neurological, psychiatric or medical illnesses such as serious cardiac arrhythmia, myo- 
cardial infarction, congestive heart failure, advanced liver disease and renal failure.

All medical records from the intensive care and general neurosurgical high dependency units were reviewed.

The serum sodium levels of each patient in the study were reviewed during the entire period in hospital or until day 9 after the cerebral aneurysm ruptured. If the serum sodium level was measured more than once on a day, then the lowest level for that day was used in this analysis. For this study, hyponatremia was defined as an absolute value of $<135 \mathrm{mmol} / \mathrm{l}$ occurring at any point in time during the first 9 days after $\mathrm{SAH}$. As part of standard protocol, the level of serum sodium was measured at least once a day in ICU and once a day in neurosurgical HDU until day 9 after SAH was over. Frequency of serum sodium measurement after day 9 was determined by previous results or possible aggravation of neurological status.

The data reviewed included:

- Demographic data like gender and age of patients.

- World Federation of Neurological Surgeons- WFNS score of neurological status on admission to hospital, information about aneurysm location and the period from rupture up to definitive treatment.

- Number of days in the intensive care unit

- Clinical deterioration due to $\mathrm{DCl}$

- Clinical outcome

Clinical deterioration due to $\mathrm{DCl}$ in medical records was diagnosed and reviewed using defined clinical criteria (focal neurological impairment, CT and CT angiography $(2,13,14,18)$.

Clinical outcome has been evaluated by the Glasgow Outcome Scale (GOS) where each patient is indicated by a corresponding number on a scale from 1 (dead) to 5 (almost complete recovery), based on data from medical records at hospital discharge (19). A Glasgow Outcome Scale (GOS) score of less than 4 defined poor outcome.

\section{Statistical analysis}

For statistical data analysis we used SPSS 13.0 (SPSS Inc. Chicago, IL). We had one group with hyponatremia and the other group without hyponatremia. Differences between the groups were analyzed by Student's t test for normal distribution data and the Mann-Whitney $U$ test for data without normal distribution. For non parametric data we used the chi-square test. The first binary enter logistic regression model evaluated the correlation of hyponatremia, WFNS grade, aneurysm size and location, rebleeding, age and gender with $\mathrm{DCl}$ and the second binary enter logistic regression analysed the influence of hyponatremia, WFNS grade, aneurysm location and size, rebleeding, age and gender on poor clinical outcome. A value $p<0.05$ was considered as statistically significant.

\section{Results}

The hospital charts of 95 patients with SAH were reviewed and 82 patients were identified for inclusion in the study. Thirty-two of the 82 patients (39\%) developed hyponatremia. None of them had serum sodium below $130 \mathrm{mmol} / \mathrm{l}$. The maximum duration of hyponatremia was 3 days. Demographic characteristics, neurological status and basic characteristics of aneurysms in both groups are presented in Table 1.

Patients in the hyponatremia group had significantly higher WFNS score on admission to hospital $(p=0.035)$.

Table 1. Demographic characteristics, neurological status and basic characteristics of aneurysms

\begin{tabular}{|c|c|c|c|}
\hline Variables & Hyponatremia & Normonatremia & $p$ value \\
\hline Age (year) & $54.2 \pm 11.3$ & $52.4 \pm 9.1$ & $p=0.015$ \\
\hline Gender M/F (n) & $10 / 22$ & $12 / 38$ & $p=0.4$ \\
\hline WFNS (n) & $2.8 \pm 1.4^{\star}$ & $2.1 \pm 1.2$ & $p=0.035$ \\
\hline $\begin{array}{l}\text { Aneurysm location } \\
\text { anterior circulation } \\
\text { posterior circulation }\end{array}$ & $\begin{array}{c}27 \\
5\end{array}$ & $\begin{array}{c}45 \\
5\end{array}$ & $p=0.5$ \\
\hline $\begin{array}{l}\text { Time of } \\
\text { intervention after } \\
\text { the rupture (day) }\end{array}$ & $2.4 \pm 3.6$ & $3.4 \pm 4.7$ & $p=0.24$ \\
\hline
\end{tabular}

Table 2. Duration of stay in ICU, clinical deterioration due to $\mathrm{DCl}$ and clinical outcome

\begin{tabular}{|lccc|}
\hline Variables & Hyponatremia & Normonatremia & $p$ value \\
\hline Duration of stay in ICU (days) & $9.5 \pm 8.2$ & $2.5 \pm 2.7$ & $p=0.001$ \\
Clinical deterioration due to DCl (patient) & yes no & yes & no \\
& 20 & 12 & 31 \\
GOS at hospital discharge $(n)$ & $3.1 \pm 1.1$ & $3.8 \pm 1.4$ \\
\hline Data are expressed as numbers or mean value \pm SD, GOS=Glasgow outcome scale & $p=0.003$ \\
\hline
\end{tabular}


Differences among the groups about duration of stay in ICU, delayed cerebral ischemia and clinical outcome are presented in Table 2.

In the normonatremia group, we had significantly fewer days in the intensive care unit after the intervention $(p=0.001)$. Cerebral vasospasm with transit or definitive deficit included 20 patients $(62 \%)$ in the hyponatremia group and 19 patients $(38 \%)$ in the normonatremia group $(p=0.03)$.

We also had a significantly better GOS level at hospital discharge in patients without hyponatremia compared to patients with hyponatremia $(p=0.003)$. In the hyponatremia group, there were 11 patients with good outcome (GOS level 4 or 5) and 21 patients with poor outcome (GOS level 1, 2 or 3). The normonatremia group included 40 patients with good outcome and 10 with poor outcome.

The binary enter logistic regression model revealed a significant correlation of hyponatremia with clinical deterioration due to $\mathrm{DCl}(\mathrm{p}=0.03 ; \mathrm{OR}=2.71 ; 95 \% \mathrm{Cl}=1.08-6.79)$ and poor clinical outcome ( $p=0.001 ; O R=7.63 ; 95 \% \mathrm{Cl}=2.77-20.88)$. This increased risk was independent of age, gender, WFNS, rebleeding, aneurysm size and location.

\section{Discussion}

Complete medical treatment, including fluid management for both groups, was carried out according to hospital SAH protocol and therefore was completely uniform for all patients. For prophylaxis of $\mathrm{DCl}$, euvolemia or mild hypervolemia was induced during the first 3 days after SAH with very similar intensity between the two groups. Control of the serum and urine osmolarity, sodium in urine and central venous pressure was a standard measure in prevention of fluid restriction and therapy guide for daily fluid intake in the case of hyponatremia. Mild hypertonic saline solution (1.5\%) was used to correct mild hyponatremia (130-134 mmol/l) if it continued for more than two days. Normal saline $(0.9 \%)$ was the only type of crystalloid used in both groups. Colloid, intermittently used for the purpose of cerebral ischemia prevention and $3 \mathrm{H}$ therapy was $6 \%$ HES 130/0.4.

The proportion of SAH patients with hyponatremia in this study is similar to data presented by Qureshi et al. and Chandy et al. $(2,3)$. The presence of a higher WFNS score as a known predictor of disease severity in the hyponatremia group was not a surprise for us. Our data correlate with the results of Sayama et al. (20), where patients with hyponatremia had a higher Hunt Hess score, but distinct from the results of Qureshi et al. where the GCS score at admission were similar between the hypo and normonatremia groups. A study by Zheng and al. (17) has positive correlations between hyponatremia and cerebral infarction but only for WFNS grade 4 or 5 patients and for hyponatremia which developed after 3 days post SAH.

The percentage of patients with $\mathrm{DCl}$ is higher than in the literature. This may be a result of the inconsistency in definition of $\mathrm{DCl}$ in different studies or a certain deficiency in our hospital SAH protocol. Data from our study about the connection of hyponatremia and symptomatic vasospasm $(\mathrm{DCl})$ is comparable with the results of Hasan et al. Widjicks et al. and Chandy et al., but distinct from the results of Qureshi et al. (2, $3,15,16)$. Charts reviewed by us did not give any definitive cause of hyponatremia, but had evidence that fluid restriction was not performed in any case.

A significantly longer duration of stay in ICU for patients with hyponatremia has revealed the perspective of more medical complications and higher hospital costs.

Observing predictive correlations between hyponatremia and poor outcome, we have found a certain connection which conflicts with the results of Qureshi et al. and Zheng et al. (17) This study has less statistical power than leading studies which we compared, but the results are still very interesting and imply a possible use of hyponatremia as an additional predictor of developing clinical deterioration due to $\mathrm{DCl}$.

We are aware that this study has several limitations. Due to the retrospective design, insufficient numbers of patients, uneven selection of patients, and data limited only to medical records, certain complications and problems could have been missed. The important observation of whether hyponatremia predates $\mathrm{DCl}$ could not be made because of lack of time precision in several records.

\section{Conclusion}

The obtained results in this study suggest certain correlation between hyponatremia, $\mathrm{DCl}$ and clinical outcome. New prospective and randomized studies are needed to determine whether strict monitoring and treatment of hyponatremia can influence on functional outcome and we are preparing performance of that type of study.

\section{Conflict of Interest}

No conflict of interest was declared by the authors.

\section{References}

1. Sherlock M, O'Sullivan E, Agha A, Behan LA, Rawluk D, Brennan P, et al. The incidence and pathophysiology of hyponatraemia after subarachnoid hemorrhage. Clin Endocrinol 2006;64:250-4. [CrossRef]

2. Qureshi Al, Suri MF, Sung GY, Straw RN, Yahia AM, Saad M, et al. Prognostic significance of hypernatremia and hyponatremia among patients with aneurysmal subarachnoid hemorrhage. Neurosurgery 2002;50:749-56. [CrossRef]

3. Chandy D, Sy R, Aronow W, Lee W N, Maguire G, Murali R. Hyponatremia and cerebrovascular spasm in aneurysmal subarachnoid hemorrhage. Neurology Ind 2006;54:273-5. [CrossRef]

4. Isotani E, Suzuki R, Tomita K, Hokari M, Monma S, Marumo F, et al. Alterations in plasma concentrations of natriuretic peptides and antidiuretic hormone after subarachnoid hemorrhage. Stroke 1994 25:2198-203. [CrossRef]

5. Revilla-Pacheco FR, Herrada-Pineda T, Loyo-Varela M, Modiano-Esquenazi M. Cerebral salt wasting syndrome in patients with aneurysmal subarachnoid hemorrhage. Neurol Res 2005;27:418-22. [CrossRef]

6. Wijdicks EF, Vermeulen M, ten Haaf JA, Hijdra A, Bakker WH, van Gijn J. Volume depletion and natriuresis in patients with ruptured intracranial aneurysm. Ann Neurol 1985;18:211-6. [CrossRef]

7. Sviri GE, Feinsod M, Soustiel JF. Brain natriuretic peptide and cerebral vasospasm in subarachnoid hemorrhage. Clinical and TCD correlations. Stroke 2000;31:118-22. [CrossRef]

8. Berendes $E$, Walter M, Cullen P, Prien T, Van Aken H, Horsthemke J, et al. Secretion of brain natriuretic peptide in patients with aneurysmal subarachnoid hemorrhage. Lancet 1997;349:245-9. [CrossRef]

9. Tomida M, Muraki M, Uemura K, Yamasaki K. Plasma concentrations of brain natriuretic peptide in patients with subarachnoid hemorrhage. Stroke 1998;29:1584-7. [CrossRef] 
10. Khurana VG, Wijdicks EF, Heublein DM, McClelland RL, Meyer FB, Piepgras DG, et al. A pilot study of dendroaspis natriuretic peptide in aneurysmal subarachnoid hemorrhage. Neurosurgery 2004;55:69-75. [CrossRef]

11. Kubo Y, Ogasawara K, Kakino S, Kashimura H, Yoshida K, Ogawa A. Cerebrospinal fluid adrenomedulin concentration correlates with hyponatremia and delayed ischemic neurological deficits after subarachnoid hemorrhage. Cerebrovasc Dis 2008;25:164-9. [CrossRef]

12. Levine JM. Critical care management of subarachnoid hemorrhage. Curr Treat Options Neurol 2009;11:126-36. [CrossRef]

13. Bederson JB, Connolly ES Jr, Batjer HH, Dacey RG, Dion JE, Diringer $\mathrm{MN}$, et al. Guidelines for the management of aneurysmal subarachnoid hemorrhage: a statement for healthcare professionals from a special writing group of the Stroke Council, American Heart Association. Stroke 2009;40:994-1025. [CrossRef]

14. Vergouwen MD, Vermeulen M, van Gijn J, Rinkel GJ, Wijdicks EF, Muizelaar JP, et al. Definition of delayed cerebral ischemia after aneurysmal subarachnoid hemorrhage as an outcome event in clinical trials and observational studies. Proposal of a Multidisciplinary Research Group. Stroke 2010;41:2391-5. [CrossRef]

15. Hasan D, Wijdicks EF, Vermeulen M. Hyponatremia is associated with cerebral ischemia in patients with aneurysmal SAH. Ann Neurol 1990;27:106-8. [CrossRef]

16. Wijdicks EF, Vermeulen M, Hijdra A, van Gijn J. Hyponatremia and cerebral infarction in patients with ruptured intracranial aneurysms: is fluid restriction harmful? Ann Neurol 1985;17:137-40. [CrossRef]

17. Zheng $B$, Qui $Y$, Jin $H$, Wang $L$, Chen $X$, Shi $C$, et al. A predictive value of hyponatremia for poor outcome and cerebral infarction in high-grade aneurysmal subarachnoid hemorrhage patients. J Neurol Neurosurg Psychiatry 2011;82:213-7. [CrossRef]

18. Chen G, Zhang JM, Hu H, Li M, Zhu KJ. Study on risk factor of severe cerebral vasospasm following aneurysmal subarachnoid hemorrhage. Chinese J Neurosurg 2005;21:665-7.

19. Jennett $B$, Bond $M$. Assessment of outcome after severe brain damage. Lancet 1975;1:480-4. [CrossRef]

20. Sayama $T$, Inamura $T$, Matsushima $T$, Inoha $S$, Inoue $T$, Fukui $M$. High incidence of hyponatremia in patients with ruptured anterior communicating artery aneurysms. Neurol Res 2000;22:151-5. 